\title{
ESTRATEGIAS DE CAZA Y PAISAJES CULTURALES EN ANTOFAGASTA DE LA SIERRA, CATAMARCA
}

\section{TI HUNTING STRATEGIES AND CULTURAL LANDSCAPES IN ANTOFAGASTA DE LA SIERRA, CATAMARCA}

\author{
Enrique Moreno \\ CONICET - Escuela de Arqueología, U.N.Ca. \\ E-mail: enalmor@gmail.com \\ Presentado el: 05/04/2013 - Aceptado 09/08/2013
}

\section{Resumen}

La categoría de paisaje es uno de los conceptos de mayor desarrollo teórico, metodológico y técnico de los últimos años dentro de la arqueología. A pesar de no ser un término de novel introducción en nuestra disciplina, la variabilidad de enfoques, así como sus potencialidades y límites han sido centrales para el desarrollo a escala global de los conocimientos sobre la interacción entre las poblaciones humanas y los ambientes en una larga secuencia histórica. Lejos estamos de pretender aquí desarrollar una prolongada y densa discusión de modelos teóricos y aplicaciones metodológicas en relación a este concepto, sino que el objetivo es evaluar su riqueza y sus alcances y posibilidades en torno a una problemática particular, como son las estrategias de caza en el paisaje de la puna catamarqueña. Sin embargo, este caso particular permite incorporar conceptos, herramientas teóricas y metodológicas que serán discutidos y puestos en práctica frente a las evidencias obtenidas. Para ello retomaremos la información obtenida de trabajos de campo realizados en la quebrada de Antofalla y en Quebrada Seca, ambos ubicados en el departamento Antofagasta de la Sierra, desde una postura del estudio del entorno entendido en términos de una articulación constante entre paisajes y seres, tanto humanos como no humanos

Palabras claves: Paisaje; Cacería; Antofagasta de la Sierra; Vicuñas

\footnotetext{
Abstract

The landscape category is one of the concepts of greatest theoretical, methodological and technical development of the last years regarding archaeology. Despite it is not a term of new introduction in our discipline, the variability of approaches as well as its potential and limits have been central for
} 
the development in global scale of the knowledge about interaction between the human populations and the environments in a long historical sequence. We are far from pretending here to develop a long and dense discussion of theoretical models and methodological applications related to this concept, being the target to evaluate its riches and its scope and its possibilities around a particular problematic, such as the hunting strategies in the landscape of the puna catamarqueña. This particular case allows us to incorporate concepts, theoretical and methodological tools, which will be discussed and implemented on the obtained evidences. In order to achieve that, we will retake the information obtained of field works performed in the quebrada de Antofalla and the Quebrada Seca, both located in the Antofagasta de la Sierra Department, from the position of the study of the landscape understood in terms of a constant articulation between landscapes and beings, both human and nonhuman.

\section{Keywords: Landscape; Hunting; Antofagasta de la Sierra; Vicuñas}

\section{Paisajes y cacería. Conceptos y comprensiones para su estudio}

No es necesario profundizar aquí que los conceptos de paisaje y de ambiente no son sinónimos, sino que al hablar de paisaje, nos referimos a las continuas, coyunturales y divergentes interacciones históricas entre poblaciones humanas, fauna, flora, relieve, hidrología, geología, clima, por nombrar solo algunos de los factores participantes. Podemos afirmar entonces que el paisaje no es algo dado, frente al cual todos los actores sociales se articulan de la misma manera. Sin embargo, esta información ha posibilitado el desarrollo de diversas posturas teóricas y metodológicas en torno de lo que ha sido denominado la arqueología del paisaje (Anschuetz et al. 2001; Bender 1993; Criado Boado 1993, 1999; Curtoni 2007; Thomas 1996, 2001; Tilley 1994). En particular, en este trabajo, la postura adoptada se basa en el paisaje entendido como un factor dinámico, en continua modificación, siendo construido, aprovechado y modificado por las poblaciones humanas, a la vez que participa en la construcción, mantenimiento o modificación de las relaciones sociales imperantes (Bender 1993; Curtoni 2007; Ingold 1993; Thomas 1996, 2001; Tilley 1994). El paisaje, por lo tanto, puede ser interpretado como un lugar creado por las personas, es decir que se encuentra impregnado de acciones y significaciones humanas. Estas acciones tienen el poder de ser reservorios de las experiencias vividas por poblaciones humanas a lo largo del tiempo. Es por ello que al circular por un paisaje determinado se corporalizan experiencias que luego son incorporadas a la memoria de los sujetos. Los cuerpos humanos se conectan con el paisaje y con los aspectos materiales presentes en ellos de manera que perduran en el tiempo perpetuando y reificando los significados culturales (Potter 2004). De esta manera “...viviendo en el paisaje, este se vuelve una parte de nosotros como nosotros nos volvemos parte de él" (Ingold 1993: 154, traducción del autor). Es decir que a la vez que vamos estructurando, preparando, viviendo el paisaje, construimos nuestra identidad en relación al paisaje, los aspectos que lo conforman y a las demás personas que lo comparten.

De esta manera el concepto de residencia, de morar un espacio cobra relevancia, ya que la relación cotidiana, la observación diaria, el cultivar los campos, el cazar algún animal, el criar los hijos, y todo tipo de práctica tiene como marco este paisaje y es en el marco de cada una de esas experiencias que se lo construye, ya que diversos aspectos del mismo remiten a experiencias vividas en el pasado. El paisaje cuenta así una historia que es cualitativa y heterogénea (Ingold 1993), o mejor dicho polisémica (Bender 1993), ya que las historias que cuenta son diversas y responden a las experiencias personales de cada agente. Así, en este discurrir cotidiano, las relaciones sociales, así como con el entorno van inscribiéndose y reproduciéndose en los cuerpos, incorporándose de esta manera a las experiencias individuales. 
De esta manera paisaje y cuerpo son conceptos complementarios que no pueden ser comprendidos independientemente (Ingold 1993). Pero además, las prácticas constituyen los paisajes en su continuo derrotero, ya que cada tarea genera una connotación tanto en el paisaje como en el cuerpo. A estas tareas que conforman y son conformadas por el paisaje, Ingold (1993) otorga el nombre de taskscape entendido como el resultado del conjunto de tareas que interactúan. A través de esta interacción, que es llevada a cabo por un agente capacitado se constituyen los actos de morar el paisaje. Es por ello que las tareas o prácticas son las que conforman el paisaje a través de la repetición de su materialización en el tiempo y en el espacio.

La arqueología posee herramientas metodológicas que nos permiten registrar tanto los ambientes actuales como reconstruir los pasados, y también identificar e interpretar las transformaciones culturales realizadas por las poblaciones humanas. Debemos ser conscientes que estas metodologías presentan una multiplicidad de límites ya que, al construirse y modificarse el paisaje rutinariamente, las evidencias identificadas remiten a momentos específicos o a prácticas particulares, las cuales son interpretadas por nosotros de acuerdo a nuestros objetivos de investigación. En el caso particular tratado aquí, nos centraremos en las prácticas de cacería, ya que la realización de esta actividad deja huellas, como ciertas estructuras construidas por los cazadores, lo que sumado a la distribución de los materiales líticos utilizados para la obtención de presas y su posterior procesamiento nos permite interpretar las características de esta práctica y el uso de los recursos obtenidos. La asociación de esta información con las características naturales del entorno y la forma en que las presas utilizan el ambiente, nos brinda un cúmulo de datos para entender la forma en que se interrelacionan cazadores, paisajes y presas para la puesta en marcha de la cacería. Quedan, por supuesto, muchas líneas todavía por evaluar, como por ejemplo, la articulación de estos paisajes cinegéticos con otras materialidades, tales como el arte rupestre, así como también las vinculaciones de otras tecnologías y saberes que se interrelacionan con esta práctica.

Pero asociado a la comprensión del paisaje propuesta más arriba, la cacería también debe ser comprendida aquí desde una perspectiva particular, ya que no implica solamente la apropiación de uno o más animales por parte de uno o más cazadores, sino que es comprendida como una práctica social, es decir, como una acción que posee connotaciones políticas, entendido este concepto en sentido amplio, es decir que pueden producir cambios intencionados o no intencionados en el ámbito social (Bourdieu 1977, 1988; Bourdieu y Wacquant 1995; Giddens 1984). Es decir, la cacería implica acciones físicas concretas, tales como la manufactura de armas, la preparación de estructuras de ocultamiento y la selección de las presas, pero a su vez, involucra acciones sociales (como por ejemplo los participantes de las partidas de caza) o religiosas (tabú de cazar ciertos animales o la prohibición de cazar algunos animales en ciertas fechas o lugares) que dan sentido a esta práctica. El problema está en segmentar una actividad dentro de diversas esferas sociales: económica, ritual, social, etc. ya que una práctica involucra y juega un rol relevante en todas estas esferas. De esta manera, los conocimientos, las experiencias o los habitus (en términos de Bourdieu 1977, 1988; Bourdieu y Wacquant 1995) implican en la interacción entre los agentes sociales, de acuerdo a relaciones de parentesco, a asociación con los antepasados, a formas de interacción con los dioses. En este sentido, toda práctica cotidiana tiene la capacidad de modificar o reproducir las relaciones sociales establecidas, y en nuestro caso particular los modos de apropiación de los recursos. 
En resumen, la cacería puede ser realizada de multiplicidad de formas diferentes, participando un número de agentes variable en cada caso, con diferentes tecnologías y modos de utilización de los recursos obtenidos. Pero lo relevante es que existe una decisión por parte de los cazadores de utilizar un espacio particular, modificarlo y realizar la cacería de un modo particular, aprovechando los recursos obtenidos también de formas disímiles. Sin embargo, en la repetición de la realización de estas prácticas, al utilizar los mismos lugares, dejando huellas perdurables en el tiempo podemos interpretar las formas en que las cacerías fueron realizadas y la relevancia de estas prácticas para las poblaciones locales a través del tiempo. En este sentido, la arqueología cuenta con las posibilidades metodológicas propicias para identificar e interpretar las huellas en el paisaje y es en ese camino en el que nos embarcaremos a continuación.

\section{El caso de Antofalla}

En diversas investigaciones realizadas en el paisaje puneño, se identificó una importancia relativa de la cacería de camélidos silvestres, más precisamente vicuñas1 en distintos contextos históricos (Aschero et al. 1991, 1993-1994; Aschero y Martínez 2001; Elkin 1996; Fernández Distel 1986; Haber 2003a, 2003b, 2006, 2007; López 2003, 2006; Martínez 2003; Núñez 1983; Núñez y Santoro 1988; Olivera 1997; Pintar 1996; Ratto 2003; Yacobaccio 1988, 2001; Yacobaccio et al. 1994, 1997-1998; Yacobaccio y Madero 1992; entre otros), que alentó el interés por estudiar las estrategias de caza implementadas por las poblaciones humanas para dar muerte a estos animales y obtener los recursos, principalmente la lana, el cuero y la carne. Para estudiar estas estrategias dos fueron las fuentes de datos principales: la tecnología de armas con las que contaban las poblaciones para dar muerte a las presas seleccionadas y los espacios utilizados para la materialización de los encuentros. En este caso focalizaremos en el último punto, pero entendiendo que en la articulación de diversos factores se pueden comprender las características particulares de esta práctica. Algunos autores han desarrollado investigaciones sobre este punto llegando a conclusiones relevantes. Tales son los casos de las propuestas de Aschero y Martínez (2001) y de Ratto (2003).

Nuestro primer caso de estudio se centra en el área del Salar de Antofalla, ubicada en el sector noroeste del Departamento Antofagasta de la Sierra, Catamarca (Figura 1). Allí, prospecciones realizadas en la cuenca de Archibarca, brindaron información relevante acerca de las características de las estrategias de cacería, en donde se articulaban paisaje, tecnología, etología de presas, a través de la construcción de dispositivos interpretados como trampas cuyo objetivo es el de poder adelantarse el cazador a los comportamientos de las presas frente al peligro que significa una potencial cacería (Haber 2003a, 2003b, 2007, 2009, Haber y Moreno 2008).

Posteriormente, la quebrada de Antofalla se presentó como un caso paradigmático para la realización de una prospección intensiva tendiente a identificar y comprender las particularidades de las estrategias de caza implementadas en dicho lugar a través de un estudio sistemático y detallado del paisaje local. En esta quebrada se articulaban algunos aspectos relevantes para el estudio de las estrategias de caza, ya que presenta factores de localización seleccionados por las vicuñas (Moreno 2010) tales como: la presencia de recurso hídrico permanente, vegas y pastizales, abrigos rocosos en sectores elevados utilizados como dormideros, laderas suaves aprovechadas por las vicuñas durante el día, y la presencia de vicuñas tanto en la actualidad como en el pasado, tal como se desprende del análisis de conjuntos faunísticos del área (Haber 2001, 2006; Moreno y Revuelta 2010; Revuelta 2005). 


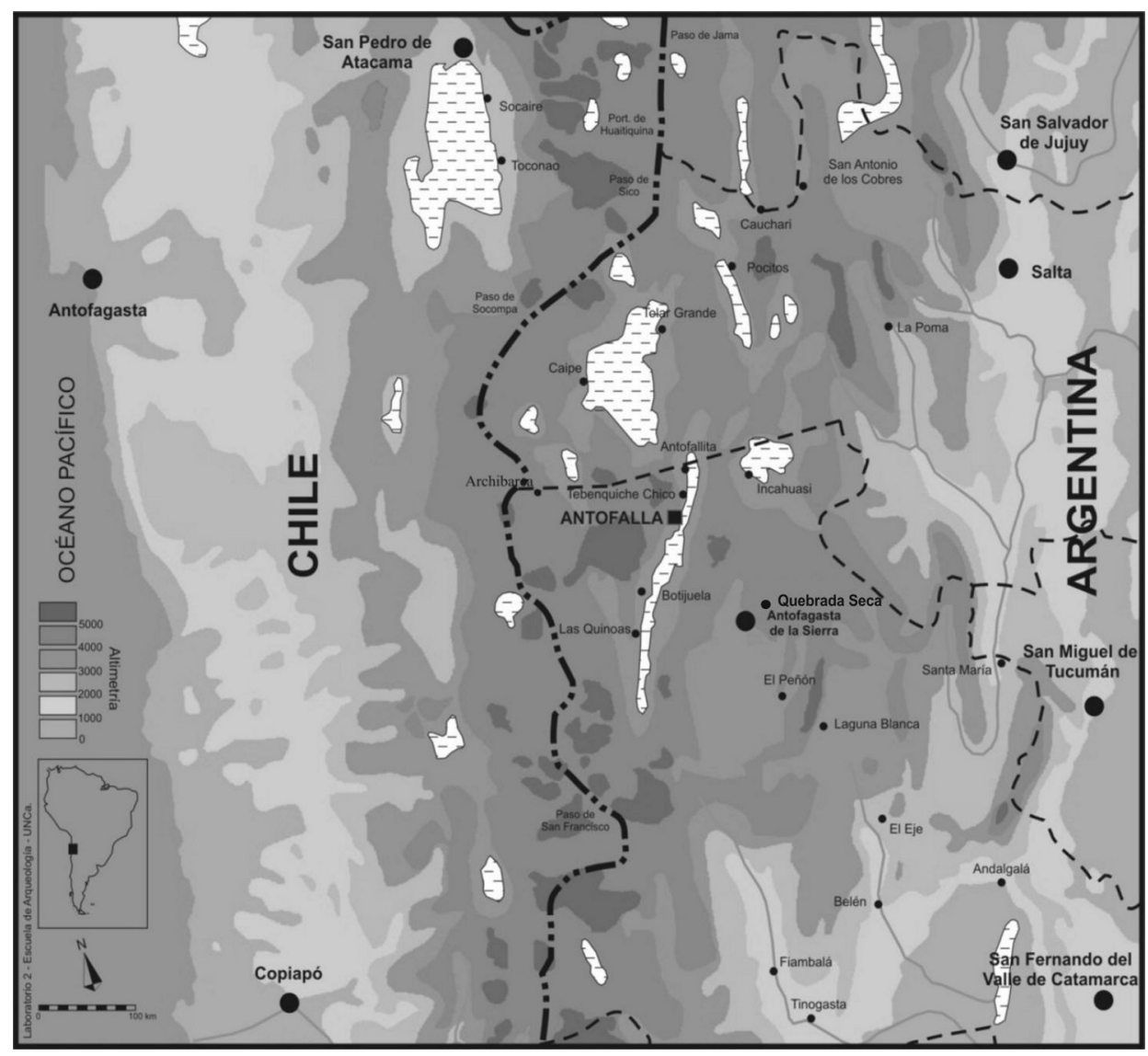

Figura 1. Mapa de ubicación de las localidades nombradas en el texto.

Asumiendo la interrelación entre teoría y metodología, los trabajos de campo se centraron en una prospección intensiva, entendida como la "observación detenida del paisaje realizada por arqueólogos con el fin de localizar restos de yacimientos pretéritos y dentro de la cual es importante, tanto o más que la recuperación de información arqueológica, el registro de las condiciones ambientales y de las características generales del terreno más allá de los meros yacimientos" (Criado Boado et al.1991: 98). De esta forma, la prospección pretende identificar no sólo las transformaciones culturales del paisaje, sino la diversidad de rasgos relevantes para reconstruir, en este caso, las estrategias de caza.

La prospección realizada en la quebrada de Antofalla abarcó un área de $40 \mathrm{~km} 2$ a través del recorrido de 660 transectas, registrándose 7675 muestreos sistemáticos de $1 \mathrm{~m} 2$ cada 100 $\mathrm{m}$ lineales recorridos (Moreno 2010). A partir de la información obtenida, identificamos una variedad de datos tendientes a la comprensión de las estrategias de caza implementadas en dicho lugar. Interpretamos a las trincheras, pequeñas estructuras en forma de media luna, de aproximadamente $1,8 \mathrm{~m}$ de largo y $0,7 \mathrm{~m}$ de alto, como las estructuras más importantes en relación al momento de encuentro entre cazadores y presas (Figura 2). Lo más significativo de estas estructuras es su ubicación en posiciones elevadas en relación a su entorno directo, es decir, permitiendo a una persona ubicada por detrás de estos pequeños muros, dominar visualmente los sectores más bajos, sin poder ser observado por las presas. Pero 
al profundizar un poco más el análisis de estas estructuras, surge relevante la asociación entre estas estructuras, asumiendo por lo tanto la posibilidad de la realización de cacerías colectivas. Tal es la magnitud de estas asociaciones que alcanzan en algunos sectores 30 trincheras en sectores acotados. El criterio seleccionado para evaluar la asociación entre estas estructuras es la posibilidad de atacar simultáneamente a una misma tropilla desde las trincheras, no existiendo barreras topográficas que eviten dicha situación (Moreno 2010, 2011b, 2012).

Pero también identificamos algunas estructuras relacionadas con momentos previos y posteriores al encuentro entre cazadores y presas y que en conjugación conforman un paisaje vivido, aprovechado, transformado y experimentado en torno a la relación entre cazadores y presas. Entre estas estructuras podemos contar refugios, mojones, alineaciones y mesadas (Moreno 2010, 2011b, 2012) (Figura 3). Todas estas aportan a la preparación, encuentro y posterior transformación de la presa en recursos varios.

De esta manera, los cazadores sumaban a las características del paisaje local estructuras de manufactura simple y rápida que permitían el escondite tanto de personas como de objetos, la marcación de áreas de caza o la delimitación de territorios. Así se configura un paisaje preparado para la realización de la cacería y que posiblemente implique la reutilización de estos espacios o por lo menos la repetición de las características básicas de la estructuración de estos paisajes.

Pero además, debemos considerar un aspecto central a la hora de analizar el paisaje cinegético como son las armas utilizadas por los cazadores para poder dar muerte a estos animales. Su relevancia se basa en que las características tecnológicas de las mismas determinan de cierta manera las posibles estrategias implementadas. Para ello la prospección fue de gran importancia, ya que además de aportar la materialidad relevante para el análisis de la tecnología, permitió la articulación con la información paisajística. En la quebrada de Antofalla identificamos 263 piezas asociadas con la manufactura de puntas de proyectil, de las cuales 71 fueron registradas como preformas mientras que las restantes 192 fueron interpretadas como fragmentos o especímenes completos de puntas de proyectil. Al tratarse de un conjunto enteramente obtenido en superficie, presenta un estado de conservación regular, identificándose sólo 13 especímenes completos de puntas de proyectil.

Para la manufactura de estos instrumentos, fueron aprovechadas materias primas locales, ya que el 65,5\% fueron elaboradas en basalto-andesita negro, proveniente de la desembocadura de la quebrada de Antofalla y el $25 \%$ en obsidiana proveniente de la cantera de Ona, ubicada a unos $15 \mathrm{~km}$. de Antofalla. El restante $10 \%$ fue manufacturado con materias primas más lejanas como el ópalo proveniente posiblemente de la cuenca de Archibarca, a unos $60 \mathrm{~km}$ al noroeste de Antofalla.

El análisis de este conjunto fue macroscópico y se centró en la morfología y la funcionalidad de los distintos especímenes (Aschero 1975, 1983). A partir de la información obtenida de este estudio (Moreno 2010, 2011a), nos centramos en la identificación de tipos morfológicos teniendo en cuenta las características tecnológicas y de diseño de estos especímenes. Dicho análisis perseguía dos objetivos: por un lado interpretar las armas utilizadas por los pobladores de Antofalla, así como también permitir la comparación con especímenes provenientes de otros paisajes semejantes y cercanos, principalmente la información obtenida en la microrregión de Antofagasta de la Sierra. 

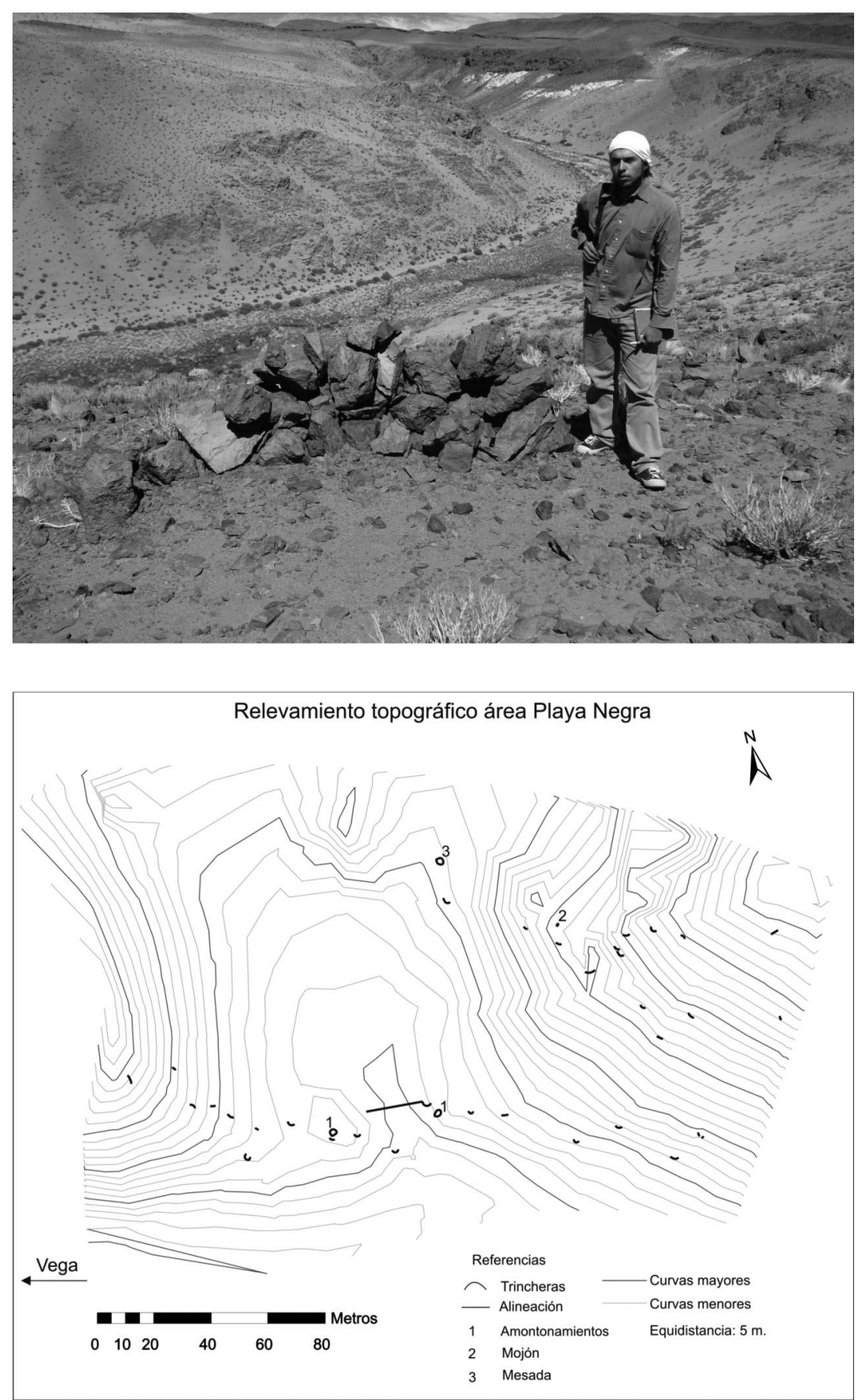

Figura 2. Arriba: ejemplo de trinchera en la quebrada de Antofalla. Abajo: Relevamiento de un conjunto de trincheras, donde se puede observar la asociación entre estas estructuras. 

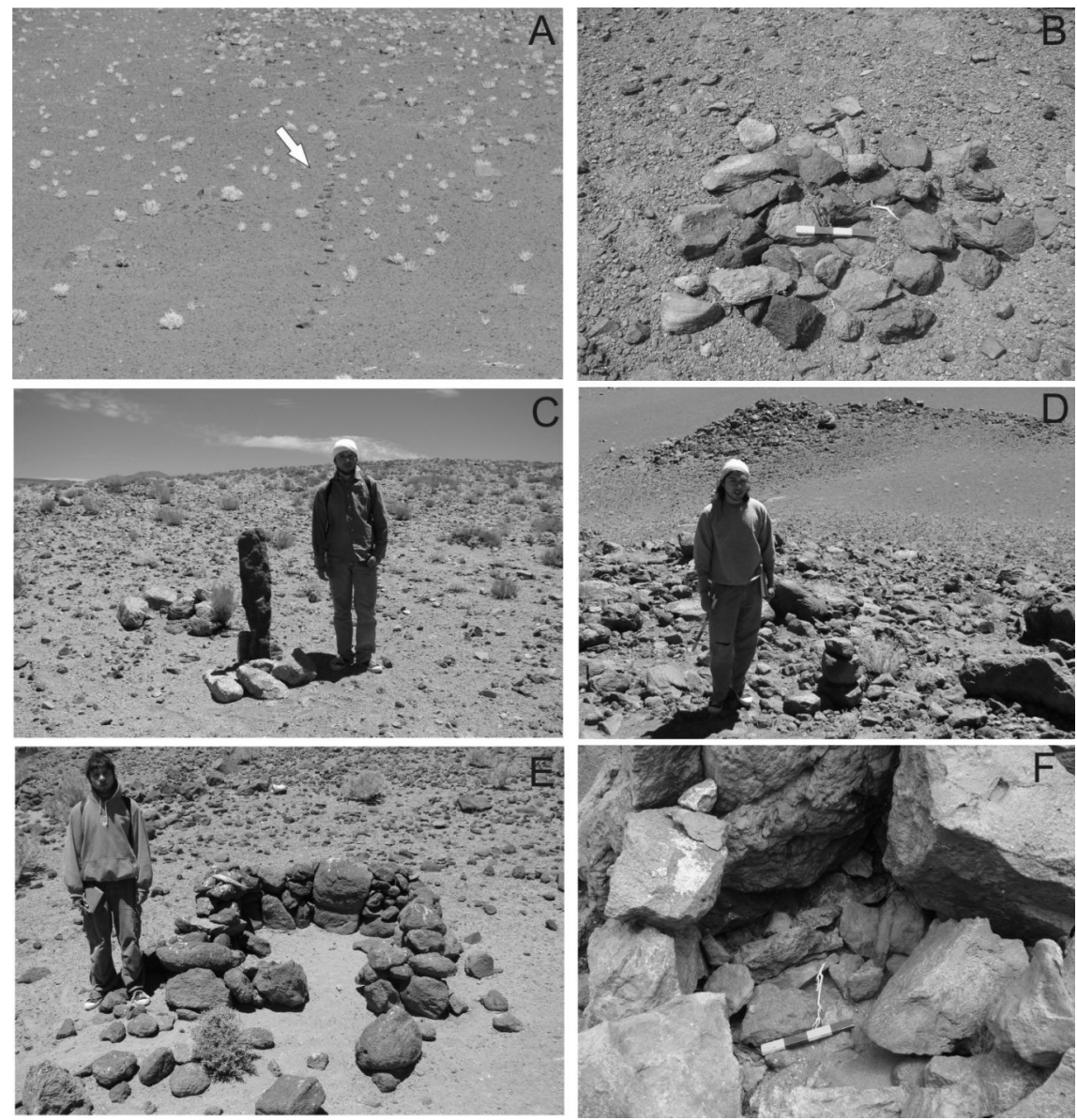

Figura 3. Estructuras identificadas en la quebrada de Antofalla. A) Alineación. La flecha indica la ubicación de esta estructura; B) Mesada; C) Mojón construida mediante la colocación de un bloque en forma vertical calzado en su base; D) Mojón construida a través de la ubicación de pequeños bloques uno encima del otro; E) Refugio;

F) Escondite de agua.

Para ello, contamos con un conjunto de 99 piezas que presentan caracteres tecnomorfológicos que permiten un análisis de este tipo, construyéndose 10 tipos morfológicos y 4 especímenes morfológicos (sensu Hocsman 2006)2 (Figura 4). Para evaluar los distintos tipos de armas utilizadas en la quebrada de Antofalla aplicamos dos métodos, por un lado la propuesta de Thomas (1978), quien basa la asignación funcional en una serie de variables métricas: largo, ancho, espesor, ancho del pedúnculo y peso. Esta metodología fue luego profundizada por Shott (1997) quien propone como variable significativa para la asignación funcional el ancho del hombro, a partir del aumento de la muestra de puntas de proyectil asignadas a dardo de lanzadera. Por su parte, Ratto $(1994,2003)$ ha generado un modelo de asignación funcional de cabezales líticos apoyándose en el análisis crítico de: (a) los equipos de caza aportados por trabajos etnográficos y experimentales, (b) la mecánica de funcionamiento de las armas en base a las leyes de la mecánica de fluidos y la trayectoria de vuelo de los proyectiles y (c) las propiedades físico-mecánicas de las materias primas líticas y vegetales. En base a estos principios se proponen una serie de aspectos relacionados a la performance del sistema técnico, tales como la superficie de refuerzo, la aerodinámica, el 
poder de penetración y las características del enmangue (Ratto 2003: 86-87). De la articulación de ambas metodologías, fue posible la identificación de 38 puntas de proyectil de las cuales diez fueron asignadas a arco y flecha, tres a armas de mano, tres a lanzas arrojadizas y veintidós a dardos de lanzadera (Moreno 2010, 2011a).

En términos generales, la información obtenida del análisis de las puntas de proyectil, nos habla del uso de distintos tipos de armas, posiblemente asociado a momentos históricos particulares, pero que en todo caso comparten un relativo bajo poder de daño y, por lo tanto, la necesidad de obtener blancos cercanos y fijos, o en caso contrario emboscados, para evitar el escape de los mismos y el fracaso de la cacería.

En este sentido, es relevante recordar brevemente algunas de las características etológicas de las presas ya que la interpretación del paisaje se cimentó fuertemente en esta información, centrándonos para ello en estudios realizados particularmente en vicuñas (Bonacic 2005;

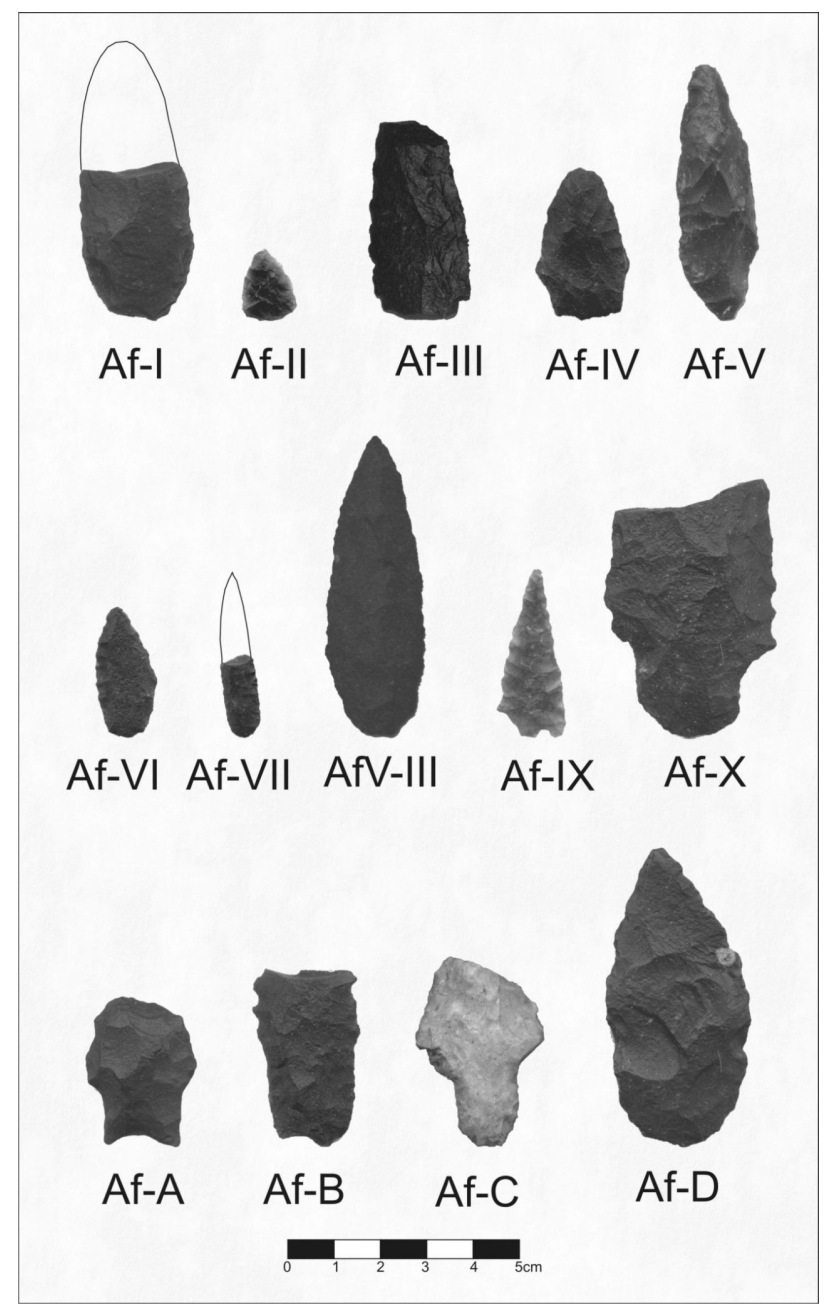

Figura 4. Tipos y especímenes morfológicos identificados en la quebrada de Antofalla. 
Franklin 1982; Hoffman et al. 1983; Koford 1957; Tomka 1992; Wheeler 2006). Uno de los tipos de agrupación identificados para esta especie, la agrupación familiar, utiliza un territorio demarcado por bosteaderos que conecta sectores elevados con roquedales aprovechados como dormideros, con sectores más bajos con agua y pasturas para alimentarse. Diariamente las tropillas, lideradas por el relincho, conectan estos sectores, descendiendo al amanecer y retornando a los dormideros al crepúsculo. El relincho dirige el movimiento de la tropilla, estando constantemente alerta y dando inicio el escape en caso de percibir cualquier posible peligro. Las vicuñas poseen la capacidad de escapar velozmente por escarpadas laderas, haciendo imposible la persecución como estrategia de cacería.

Tomando en consideración lo anterior, la construcción de las estructuras descriptas más arriba, permiten a los cazadores - conocedores de los comportamientos de sus presas - ubicarse en sectores elevados, localizados en posiciones intermedias entre los comederos y los dormideros, anticipándose de esta manera a los movimientos de las presas, pudiendo sorprender a los animales en su ascenso y de esta manera reducir sus ventajas adaptativas (velocidad, percepción de ruidos y olores, etc.) y las limitaciones de la tecnología de caza con la que contaban estas poblaciones. Al intersectar las diferentes fuentes de información, proponemos la realización de dos posibles estrategias de caza: por un lado la de espera, es decir, el/los cazador/es escondido detrás de las trincheras aguardando que la tropilla circule por los espacios por los que rutinariamente transitan para realizar la cacería o también la participación de azuzadores que forzaran a las vicuñas a dirigirse hacia los sectores donde esperaban los cazadores parapetados. La ventaja de la primera estrategia era la posibilidad de obtener blancos cercanos y prácticamente inmóviles, pero con una alta posibilidad que la tropilla ascienda por otro lugar implicando el fracaso de la cacería. La segunda estrategia obliga a los animales a transitar hacia los cazadores, pero en estampida, escapando, por lo que los cazadores tendrían blancos en movimiento, lo que dificultaría hacer blanco en ellos. Sin embargo, ambas estrategias se apoyan en la posibilidad de disminuir las capacidades adaptativas de las vicuñas para poder aumentar las chances de éxito de la cacería. Estas estrategias sumadas a la participación de varios cazadores, habrían aumentado dichas chances.

Un aspecto importante de la estructuración de este paisaje es la reutilización de los espacios y de las estructuras de cacería. Para evaluar este aspecto, el diseño de las puntas de proyectil nos otorga información para la construcción de una cronología relativa del uso de estos paisajes. El mismo se basa en la comparación con especímenes que han sido recuperados en contextos con fechados radiocarbónicos del NOA y del norte chileno (De Souza 2004; Escola 1987, 2000; Hocsman 2006; Martínez 2003; Núñez 1983; Ratto 2003). En la Figura 5 presentamos los resultados de esta comparación que muestra diseños correspondientes a diferentes momentos históricos, desde el 8000 AP hasta el primer milenio de la era. Pero además contamos con información sobre las armas utilizadas en contextos históricos más tardíos, como son puntas de proyectil manufacturadas en material óseo y cartuchos de armas de fuego (Haber 2006; Lema 2004; Moreno 2010).

Pero aquí debemos hacer varias observaciones acerca de la escala temporal de esta práctica. Quizás uno de los principales límites de la arqueología del paisaje sea el establecimiento de cronologías para poder ubicar en el tiempo las transformaciones del paisaje que observamos. Es complejo poder ubicar temporalmente las estructuras y materiales arqueológicos identificados. En el caso particular de la quebrada de Antofalla, entendemos que el análisis aquí realizado corresponde a la expansión máxima del paisaje cinegético, que 


\begin{tabular}{|c|c|c|}
\hline Tipo & Semejanza & Período \\
\hline Af-I & Peñas Chicas E & $4150-3430 \mathrm{AP}$ \\
\hline Af-II & Quebrada Seca A - Tambillo-1 & $8600 \mathrm{AP}$ \\
\hline Af-III & X & X \\
\hline Af-IV & Peñas Chicas C & $4150-3430 \mathrm{AP}$ \\
\hline Af-V & X & X \\
\hline Af-VI & Quebrada Seca F & $4150-3430 \mathrm{AP}$ \\
\hline Af-VII & Peña de la Cruz A & 7270 AP \\
\hline Af-VIII & Peñas Chicas A & $4150-3430 \mathrm{AP}$ \\
\hline & $\begin{array}{c}\text { Registros semejantes en TC1, } \\
\text { Casa Chavez Montículos, }\end{array}$ \\
Chaschuil, Real Grande I, Tulan & \\
Af-IX & 54 & $1^{\circ}$ Milenio d.C. \\
\hline Af-X & X & X \\
\hline Af-A & Quebrada Seca B & $7350-3500$ AP \\
\hline Af-B & X & X \\
\hline Af-C & X & X \\
\hline Af-D & Peñas Chicas 4 & 4150 - 3430 AP \\
\hline
\end{tabular}

Figura 5. Cuadro comparativo de los diseños identificados en la quebrada de Antofalla, con especímenes de otras regiones cercanas como Antofagasta de la Sierra o el Norte Chileno y su posible asignación cronológica.

supone a su vez, la adición no planificada y durante un largo término histórico de los distintos tipos de estructuras, siendo posible la utilización de algunos espacios una sola vez, mientras que otros espacios pudieron ser reutilizados. Ha sido imposible secuenciar la construcción de estos espacios, ya que la manufactura simple de las estructuras, así como su ubicación en lugares de relativamente poco tránsito y transformación en el tiempo han permitido una conservación homogénea. Tampoco pudimos identificar diferencias constructivas, salvo las que fueron asociadas a su funcionalidad y a su ubicación topográfica.

Siendo conscientes de esta situación, es posible apuntar algunas claves que en la articulación de la información arqueológica obtenida aportan resultados relevantes. Así, hemos interpretado que el sector más bajo de la quebrada, hasta su desembocadura, no habría sido utilizado para la realización de cacerías durante los últimos dos milenios, ya que dicho espacio fue aprovechado para actividades agrícolas (Quesada 2007) que implicarían la constante circulación de personas regando, cultivando y cosechando, lo que haría que no fuera un área seleccionada por las tropillas de vicuñas para establecer su territorio, ya que la presencia de humanos encerraría un peligro constante. Si a esto sumamos la información de la relevancia de la cacería de vicuñas en estos contextos (Haber 2001, 2006; Moreno 2010; Moreno y Revuelta 2010; Olivera 1997; Revuelta 2005), podemos suponer que algunos otros espacios de la quebrada, como Playa Negra, o las áreas más elevadas, cercanas al Ojo de Agua, habrían sido utilizados para la materialización de esta práctica, ya que se encontraban alejadas de los sectores aprovechados para la agricultura y presentan condiciones buscadas por las vicuñas para establecer sus territorios (Figura 6). En este sentido, pensamos que la ubicación de las puntas de proyectil de acuerdo a su asignación cronológica descripta más arriba, podría aportar cierta información sobre este interrogante. Sin embargo, dicho análisis muestra una distribución relativamente homogénea de los especímenes de puntas de proyectil asignados a los diferentes contextos históricos. Se observa una mayor presencia de piezas asignadas 


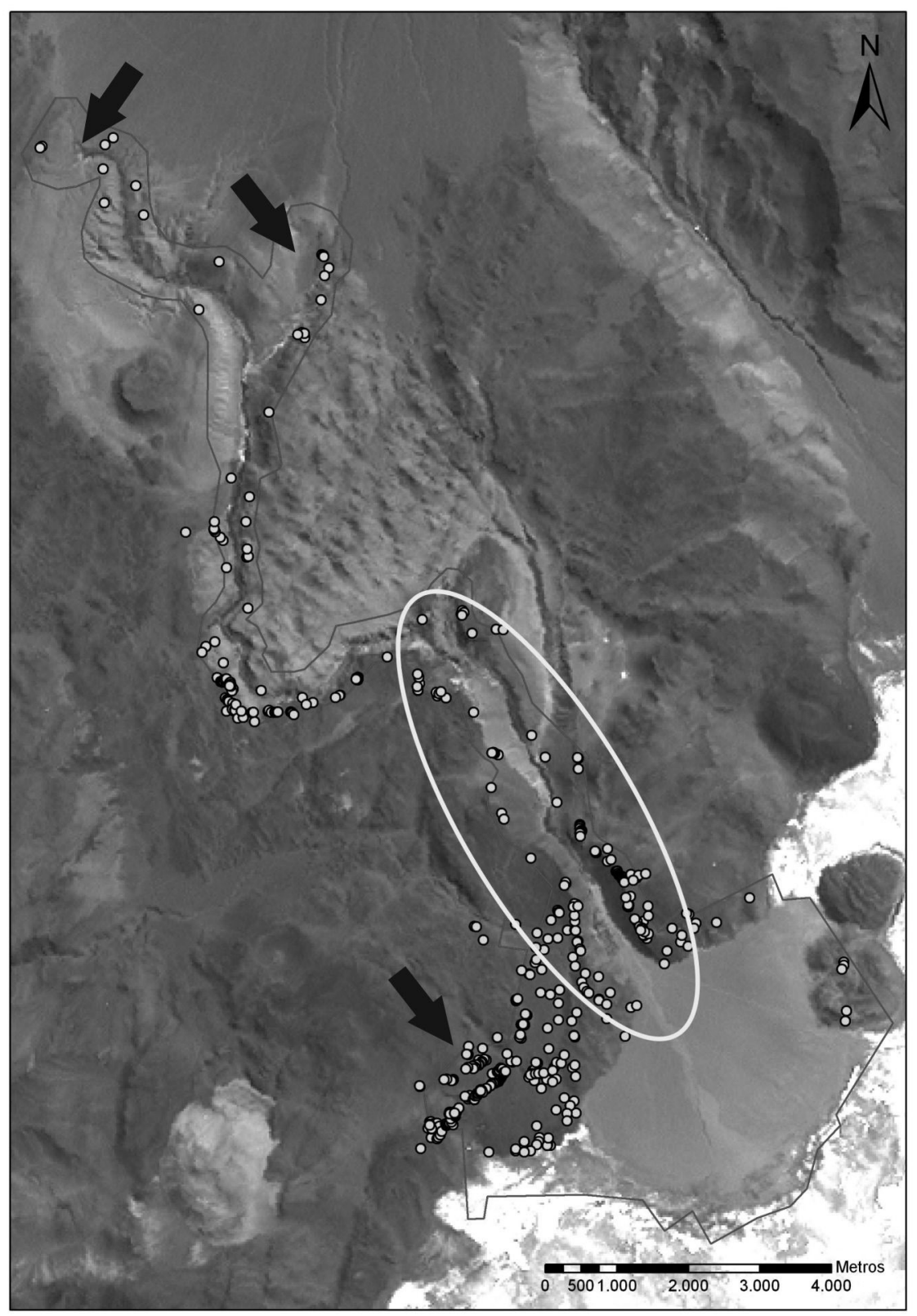

Figura 6 Distribución de las trincheras en la quebrada de Antofalla. El ovalo indica el área bajo producción agrícola durante el $1^{\circ}$ y $2^{\circ}$ milenio de la era. Las flechas indican la ubicación de Playa Negra y de los ojos de agua que dan origen a la quebrada.

al denominado período de transición, pero al analizar en términos porcentuales, vemos que hay una recurrencia en la ubicación de los distintos diseños de puntas de proyectil en los mismos espacios. Esto nos permitió pensar en la reutilización recurrente de ciertos espacios para la realización de cacerías, en donde los factores de localización buscados por las potenciales presas se encuentran presentes y que por lo tanto habrían posibilitado la presencia de animales, aumentando las posibilidades de la realización de cacerías. 
Pero aquí estamos hablando de una escala temporal de gran escala, que se basa en la cronología relativa al uso de estos paisajes y en su posible reutilización. Pero ya en otras ocasiones (Haber y Moreno 2008; , Moreno 2010, 2011b, 2012) hemos planteado que la ubicación topográfica de estas estructuras nos permiten interpretar el momento del encuentro entre animales y cazadores, que se habría realizado al atardecer, en el crepúsculo, momento en el cual los animales, regresando a los sectores aprovechados como dormideros, eran atacados por los cazadores, parapetados detrás de las trincheras, generando un ataque sorpresivo y posiblemente certero. Previo al momento del encuentro, el o los cazadores debían seleccionar, de acuerdo a la observación de las tropillas, el lugar propicio para realizar el ataque.

Pero también al realizarse la cacería en este horario era posible el manejo de otros factores, como por ejemplo la luz solar. Identificamos una mayor cantidad de trincheras sobre la ladera oeste de la quebrada, lo que provocaría que al ascender por la ladera, las posibles presas tendrían el sol de frente, haciendo más dificultosa la percepción de los cazadores ya escondidos detrás de los pequeños muros, listos para realizar el ataque. También al atardecer, los vientos suelen direccionarse hacia la desembocadura de la quebrada, originándose en sectores más altos. Esto haría que los olores de los cazadores no fueron percibidos por las presas, acercándose a las trincheras sin percibir el peligro.

Así en la quebrada de Antofalla, pudimos identificar una serie de transformaciones del paisaje tendientes a la necesidad de los cazadores de anticiparse a los movimientos de las posibles presas y de esta forma disminuir las capacidad de percibir el peligro y escapar que poseen las vicuñas y a la vez obtener blancos lo más cercanos posibles. Sin embargo, esto no impediría la utilización de estrategias de acarreo para asegurar el paso de las tropillas por los espacios deseados. La posible desventaja de dicha estrategia sería que los animales podrían transitar velozmente, escapando del peligro, haciendo que el blanco fuera más difícil de alcanzar con la tecnología con la que contaban estas poblaciones.

Pero ahora bien, la quebrada de Antofalla, tal como hemos mostrado hasta aquí, posee ciertas características que la convierten en un caso paradigmático y con un volumen de información muy importante, que nos llevó a preguntarnos si se podría observar el mismo tipo de paisaje en otros lugares y permitir la comparación en términos de las estrategias implementadas por los cazadores, aplicando las mismas herramientas metodológicas descriptas más arriba. Para evaluar este interrogante, realizamos una prospección en la localidad de Quebrada Seca, ubicada a unos $15 \mathrm{~km}$. de la localidad de Antofagasta de la Sierra y con un importante cúmulo de información sobre ocupaciones humanas durante los últimos 10.000 años.

\section{El caso de Quebrada Seca}

Quebrada Seca se encuentra a unos $15 \mathrm{~km}$ al este de la villa de Antofagasta de la Sierra, a una altura sobre el nivel del mar de $4100 \mathrm{~m}$. Presenta una longitud de $7 \mathrm{~km}$ y se encuentra surcada por cursos de agua y sectores de vega que se alternan con tramos secos. La quebrada presenta laderas escarpadas formadas por altos farallones de depósitos ignimbríticos tabulares grises y taludes de detritos (Elkin 1996). Estas características de la quebrada son de determinante importancia a la hora de interpretar las particularidades de las ocupaciones humanas, sobre lo cual volveremos más adelante. 
Esta quebrada es especialmente conocida por el alero Quebrada Seca 3 (QS3) excavado durante la década del 80 y 90 por Carlos Aschero y equipo. Dichas excavaciones, sumadas a informaciones de otros sitios cercanos permitió construir una secuencia de ocupación humana de larga duración en la zona, donde una de las principales actividades económicas era la cacería de camélidos, especialmente de vicuñas (Aschero et al. 1991, 1993-94; Elkin 1992, 1996; Hocsman 2006; Martínez 2003; Rodríguez 1998).

Sobre la base de esta información, planeamos una prospección intensiva desarrollando un diseño que nos permitiera muestrear los distintos sectores de la quebrada, incluyendo las planicies de altura, pudiendo de esta manera contrastar esta información con la obtenida en la quebrada de Antofalla.

Sin embargo, una vez en el campo, una serie de obstáculos hicieron imposible la realización de dicho diseño, implicando la necesidad de generar una estrategia de prospección in situ. El inconveniente más importante lo constituyen las escarpadas laderas que unen el fondo de la quebrada con la pampa alta, que en algunos casos alcanzan los 100 $\mathrm{m}$. de altura, sumado a la presencia de los farallones que imposibilitan en largos tramos el tránsito entre ambos sectores. Vale aclarar que este paisaje habría negado el tránsito tanto de las vicuñas como de los cazadores, por lo que entendemos que realizar una prospección en estos sectores no habría otorgado información alguna (Figura 7). Este es un punto de fuerte diferencia entre las quebradas analizadas en este trabajo.

Otra característica del paisaje local que nos hizo modificar el plan original fue la presencia de extensas pampas altas, las cuales fueron recorridas durante los primeros días

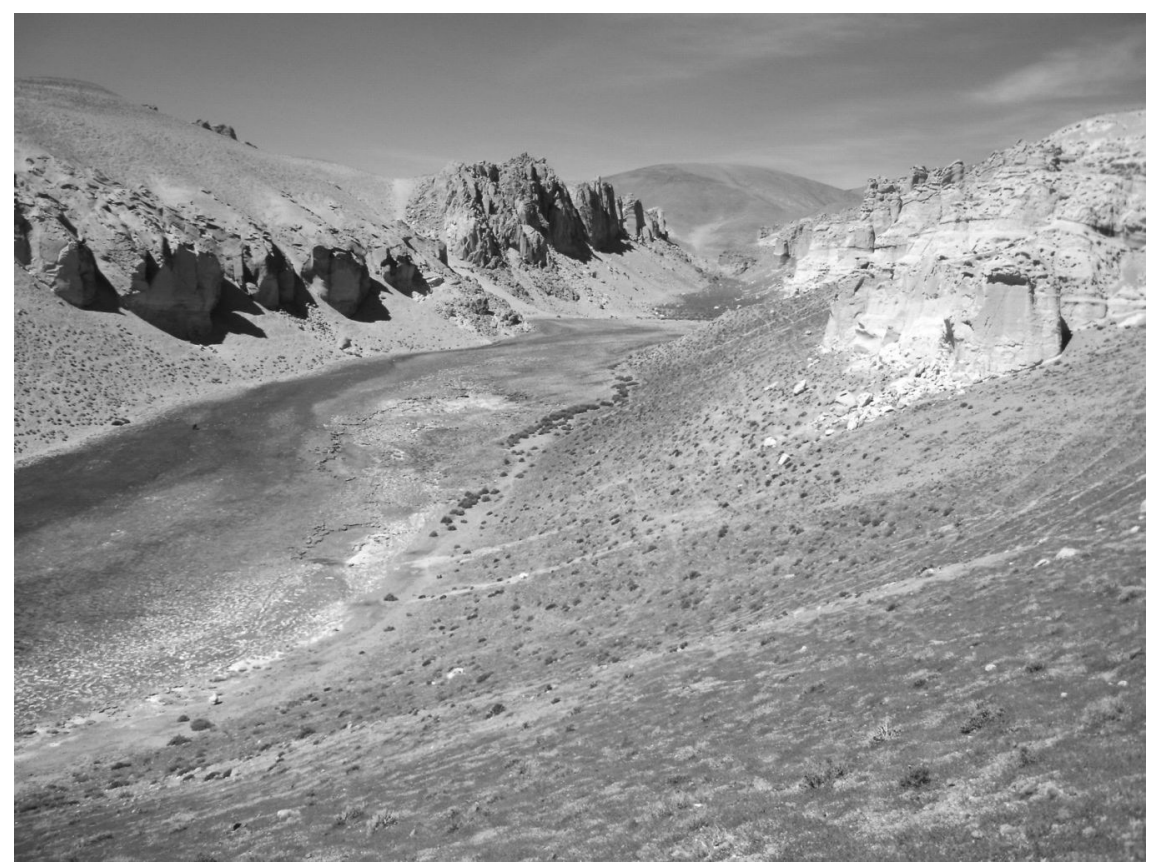

Figura 7. Vista de un sector de Quebrada Seca donde se puede observar la pendiente de las laderas, así como también la presencia de bloques ignimbríticos. 
de prospección con una tasa de hallazgo extremadamente baja, lo que implicaba un muy alto costo de energía y tiempo para recorrerlas y con una cantidad de información escueta, por lo que decidimos enfocarnos a otras áreas de la quebrada, considerando la potencialidad de su comparación con la información obtenida en Antofalla.

Finalmente el tercer aspecto relevante fue la asociación de los hallazgos al recurso hídrico. Es claro y ha sido postulado repetidas veces que en ambientes como el puneño un recurso clave es el agua, probablemente el más relevante. Al realizar un primer acercamiento a Quebrada Seca pudimos observar que este recurso se encuentra acotado a un sector de la misma, interpretándose por lo tanto que el área a prospectar debía corresponderse con la presencia de este recurso, ampliándose así el área de prospección donde se encuentra el agua y reduciéndose en otros sectores.

Por lo tanto, teniendo en cuenta el objetivo de recorrer la mayor cantidad de terreno, aumentando la probabilidad de hallazgos en un acotado período de tiempo, diseñamos transectas que se adecuaran de mejor manera a este objetivo.

En total se desarrollaron 30 transectas, además de recorridos dirigidos hacia sectores con un potencial alto de hallazgos arqueológicos, los cuales no fueron recorridos utilizando esta metodología3. Uniendo estos aspectos, el área recorrida abarcó un poco más de $5 \mathrm{~km} 2$ identificando un total de 122 unidades de registro (Figura 8).

$\mathrm{Al}$ igual que en el caso de Antofalla, dichas unidades de registro fueron diferenciadas en estructuras, hallazgos aislados y dispersiones de material superficial. En el caso de las estructuras fue posible identificar un total de 67, de las cuales 14 fueron interpretadas como estructuras actuales, asociadas al encierro de animales y a la delimitación de áreas de pastoreo. Las restantes 53 estructuras, han sido interpretadas como prehispánicas, por sus características arquitectónicas y su asociación con material superficial, principalmente material lítico tallado.

Considerando el objetivo de este trabajo, es decir las estrategias de caza implementadas en el paisaje puneño a través de la estructuración del paisaje cinegético, las estructuras más frecuentemente identificadas son las trincheras, registrándose un total de 24 estructuras. $\mathrm{Si}$ consideramos el número de estructuras en relación al área prospectada podríamos pensar que este representa un número importante de este tipo de estructuras, sobre todo considerando los amplios sectores de pampa de altura que fueron prospectados, donde inferimos que no se habrían realizado cacerías. Sin embargo, de las 24 trincheras, 20 se encuentran asociadas en un mismo sector, conformado por un afloramiento rocoso que se ubica en dirección este-oeste y que comunica a través de un cañadón prolongado el fondo de la quebrada, con presencia de agua y pasturas, con el sector más elevado, donde las tropillas buscarían refugio al atardecer en los afloramientos rocosos (Figura 9). El resto de las trincheras, se ubican aisladas, pero repitiendo las características identificadas en Antofalla, es decir, ubicadas en sectores elevados con buena visibilidad y en posiciones intermedias entre los comederos y los dormideros.

Este conjunto de 20 estructuras puede ser interpretado en los mismos términos que aquellos conjuntos registrados en la quebrada de Antofalla. Presenta una asociación directa entre estructuras de muy similar manufactura, en un sector elevado que les otorgaría a los cazadores un buen escondite y además en un espacio intermedio entre las áreas de pastura 


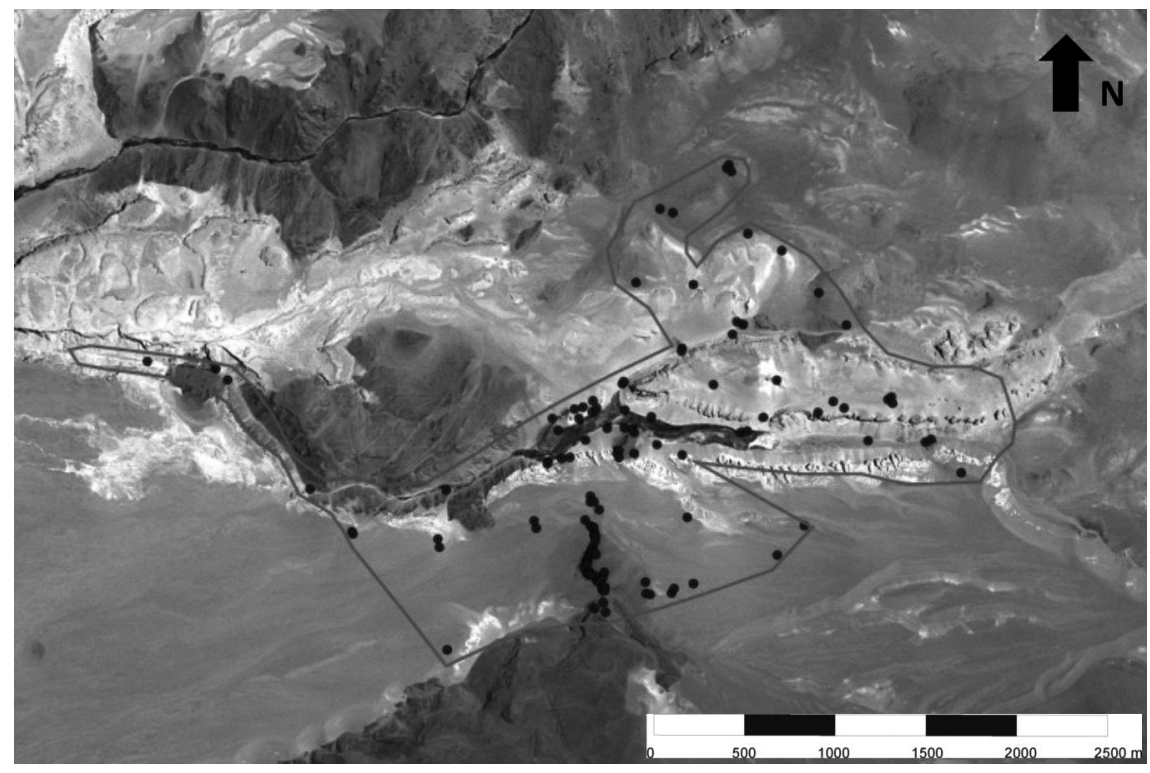

Figura 8. Límite de la prospección y unidades de registro identificadas en Quebrada Seca.

y los sectores elevados posiblemente aprovechados como dormideros. Sin embargo, esta asociación de estructuras presenta algunas diferencias con lo que pudimos observar e interpretar para el caso de Antofalla.

En primer lugar, se trata de un afloramiento rocoso asociado a una planicie de altura, que haría plausible que las vicuñas escaparan fácilmente o se alejaran lo suficiente para hacer prácticamente imposible el ataque, ya que las trincheras se encuentran ubicadas sobre la ladera a una distancia considerable, aproximadamente de 15 a 25 metros, del quiebre de pendiente inferior del afloramiento.

En segundo lugar, se ubican elevadas en relación al entorno directo, pero no comparte con el caso de Antofalla la ubicación en el quiebre de pendiente superior y aprovechando espacios mucho más confinados que hagan relativamente más complicado el escape de estos animales.

Por último, y a nivel más general, en Quebrada Seca sólo registramos esta única asociación de estructuras, mientras que en Antofalla prácticamente la totalidad de los quiebres de pendientes presentaban estructuras de este tipo, con un alto porcentaje de asociación entre estructuras (Moreno 2010, 2011b, 2012).

En síntesis, ambas quebradas presentan diferencias y semejanzas significativas, teniendo en cuenta la diferencia en la escala de ambas prospecciones. Esto podría implicar la implementación de diferentes estrategias de caza o una diferencia en cuanto a la relevancia de la cacería entre ambos lugares, considerando en Antofalla una gran importancia de esta práctica, mientras que en Quebrada Seca, un valor relativamente bajo. 

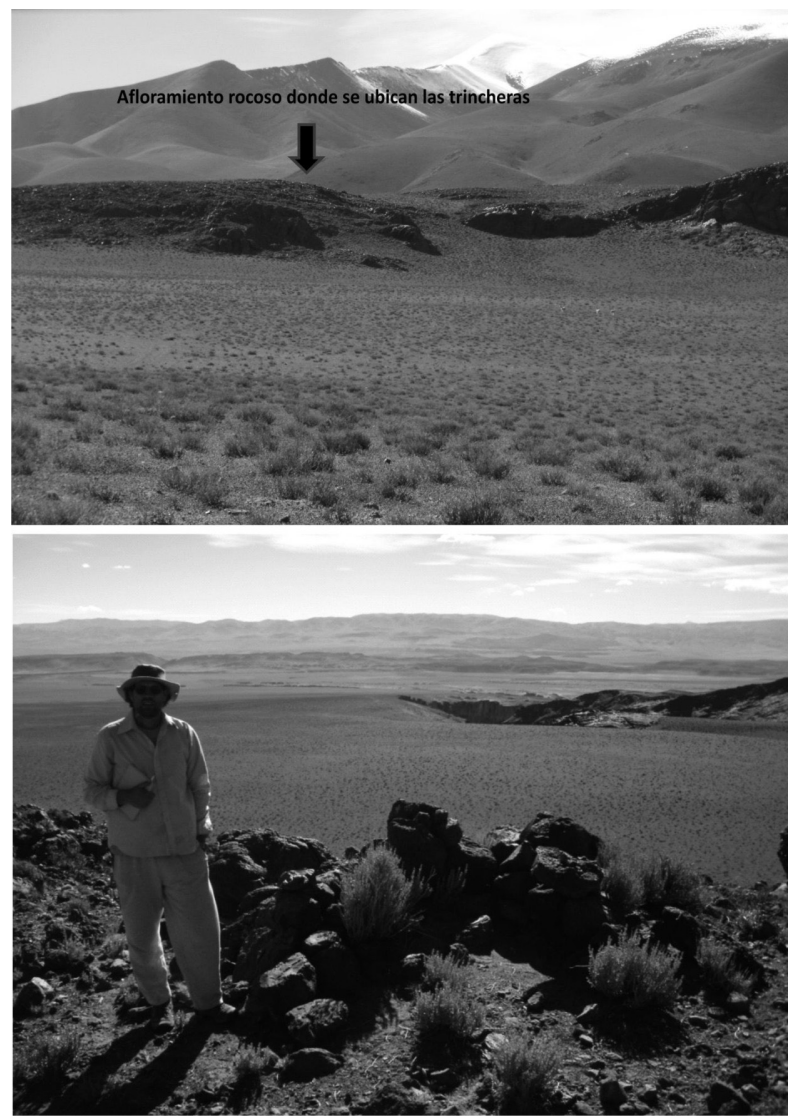

Figura 9. Vista general del afloramiento donde fue identificado el conjunto de trincheras. Nótese la relación del afloramiento con la pampa de altura. Abajo: ejemplo de trinchera.

Pero es claro que en Quebrada Seca la cacería de camélidos silvestres fue una de las principales prácticas de reproducción social, tal como lo muestran las conclusiones arrojadas por los análisis de conjuntos faunísticos (Aschero et al. 1991; Aschero y Martínez 2001; Elkin 1992, 1996; Olivera y Elkin 1994). En contraposición en Antofalla interpretamos la relevancia de las prácticas de caza a partir del importante desarrollo del paisaje cinegético y de la información obtenida de conjuntos faunísticos e instrumental lítico de las excavaciones realizadas en la quebrada de Tebenquiche Chico (Haber 2001, 2006; Moreno y Revuelta 2010; Revuelta 2005).

Por lo tanto, si la cacería fue una importante práctica en ambos lugares, ¿las diferencias responden a estrategias de caza alternativas?

En primera instancia, el paisaje y los rasgos particulares apuntan algunas consideraciones importantes. A pesar que ambos casos de estudios son quebradas, esto dista de presentar escenarios estrictamente homogéneos. En el caso de Antofalla, el recurso hídrico es constante y superficial desde los ojos de agua principales, hasta la desembocadura en el Salar, mientras que en el caso de Quebrada Seca se encuentra acotado a ciertos sectores, haciendo que los recursos se acumulen en estas áreas. Esto pudo ser verificado en la distribución de las 
unidades de registro identificadas durante la prospección que se encuentran altamente determinadas a las áreas con presencia de este recurso. También las escarpadas y abruptas laderas, así como la presencia de bloques graníticos e ignimbríticos impiden la articulación entre los sectores bajos de la quebrada utilizados para alimentarse por parte de las tropillas y las áreas elevadas donde los animales buscaban refugio para pernoctar (Figura 10). Esta conexión, sin embargo, podía realizarse a través de prolongados cañadones, que presenta grandes bloques en las áreas elevadas que podrían cumplir el rol de dormidero para las vicuñas. Esta situación provocaría ciertas diferencias en las estrategias de caza debido a que no todos los espacios podrían ser utilizados para la cacería, ya que no serían paisajes por los que circularían las tropillas. De esta manera, la opción sería fortalecer el direccionamiento de los animales a través de la utilización de azuzadores y aprovechar estos grandes bloques para esconderse, utilizándose como 'parapetos naturales' tendientes a esconderse de las presas y materializar el ataque. En contraposición, en Antofalla, las características del relieve, con espacios casi continuos de conexión entre vega y quiebres de pendiente superiores, permitían la reproducción del paisaje de caza y de las estrategias de espera para realizar el ataque, pudiéndose aprovechar prácticamente la totalidad de la extensión de la quebrada y por lo tanto la reproducción en la quebrada de la misma lógica espacial.

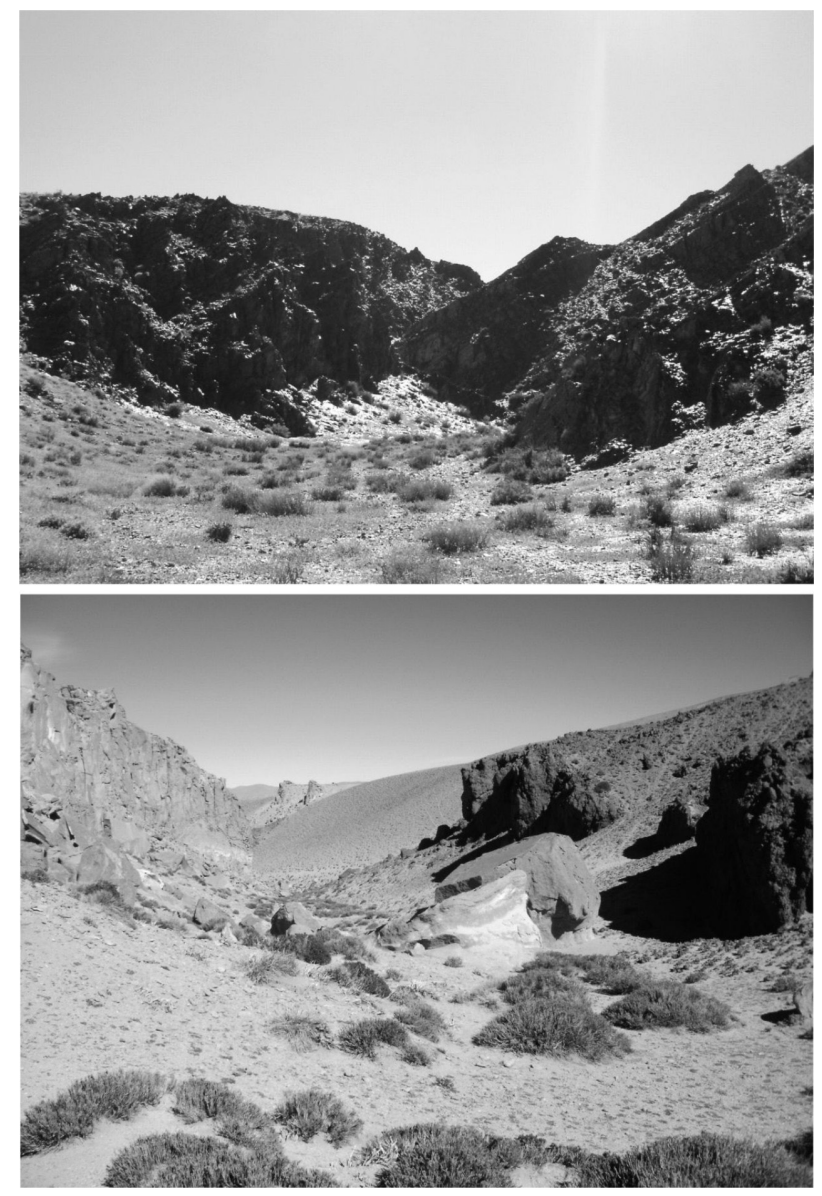

Figura 10. Cañadones registrados en Quebrada Seca. En la figura de abajo se pueden observar grandes bloques que podrían ser utilizados para el escondite. 
Otro aspecto relevante es que la concentración del recurso hídrico en el caso de Quebrada Seca concentraría la presencia humana, ya que en dicho sector, además de la presencia de agua apta para el consumo, se suma el refugio aportado por aleros, como es el caso de QS3, pudiendo esta situación generar el escape de las vicuñas hacia otros espacios, haciendo que la cacería se realizara en otro lugar.

Hasta aquí nos hemos centrado en la comparación de las características del paisaje. Pero qué sucede con la tecnología de caza. ¿Existen diferencias entre ambas quebradas? Durante la prospección en Quebrada Seca registramos un total de 16 fragmentos y piezas enteras de puntas de proyectil, notándose el aprovechamiento principalmente de vulcanitas 2, 5, 6 y 7 cuyas canteras se ubican en sectores próximos a Quebrada Seca y en obsidiana proveniente de Ona, ubicada a unos $90 \mathrm{~km}$ de distancia (Figura 11). Resalta del análisis de este conjunto los diseños correspondientes a diferentes momentos históricos, desde diseños asignables al período temprano, como aquellos correspondientes al período de transición. Pero además si consideramos la información obtenida de las puntas de proyectil provenientes de la excavación de QS3 observamos una variabilidad de diseños de puntas de proyectil muy alta que reflejaría diferencias en los sistemas de armas del cual habrían formado la parte activa (Aschero y Martínez 2001; Hocsman 2006; Martínez 2003).

Estas diferencias en los diseños, específicamente para el momento que se corresponde entre el 7000 y el 10000 AP fue correlacionado por Aschero y Martínez (2001) con diferentes estrategias de cacería. Uno de estos modelos (caza en espacios abiertos $8660 \pm 80$ AP y $8640 \pm 60$ $\mathrm{AP})$ sería aquel implementado mediante la cacería con el uso de propulsor mediante la aplicación del acecho o persecución, sin arreo. El segundo modelo (caza por intercepción) implicaría en un primer caso la utilización de azuzadores para direccionar las tropillas hacia angostos o cañadones donde los cazadores, munidos de propulsores realizarían el ataque. En el segundo caso, el arma sería la lanza arrojadiza que requiere la reducción de la distancia a las presas y el mejoramiento de las condiciones de ocultamiento, haciendo necesaria la construcción de parapetos. Finalmente se propone la caza por acecho con el uso de propulsores, donde se construye un número importante de parapetos, asociados a rasgos topográficos relevantes, tal como el caso descripto más arriba.

Si evaluamos brevemente estos modelos, notamos que en el primer caso, el modelo fue construido a partir de la ausencia de puntas de proyectil correspondientes a dicho período histórico, por lo que asumen la realización de cacerías en pampas o vegas. Sin embargo, considerando la etología de las presas, entendemos que este tipo de cacerías resultaría en una muy baja tasa de éxito, ya que obtener ocultamiento y poder acercarse a distancias de tiro de las tropillas se volvería una tarea poco probable. Los otros dos modelos, a pesar de presentar diferencias relevantes, muestran la articulación entre rasgos topográficos, la construcción de parapetos y la posible circulación de las vicuñas, aunque hacen un hincapié bastante fuerte en la participación de azuzadores. El criterio utilizado para la interpretación de estos diferentes modelos es la asociación de puntas de proyectil de distintos diseños en sectores diferentes de la quebrada.

Sin embargo podemos sugerir que la asociación entre distintos tipos de puntas de proyectil y estrategias de caza requería de la implementación de la evaluación de las características del paisaje para apoyarlas o discutirlas. En contraposición, en Antofalla, las investigaciones mostraron la reproducción de una estrategia de caza común a través del tiempo, diferenciándose factores específicos, pero no la estrategia en términos generales. 


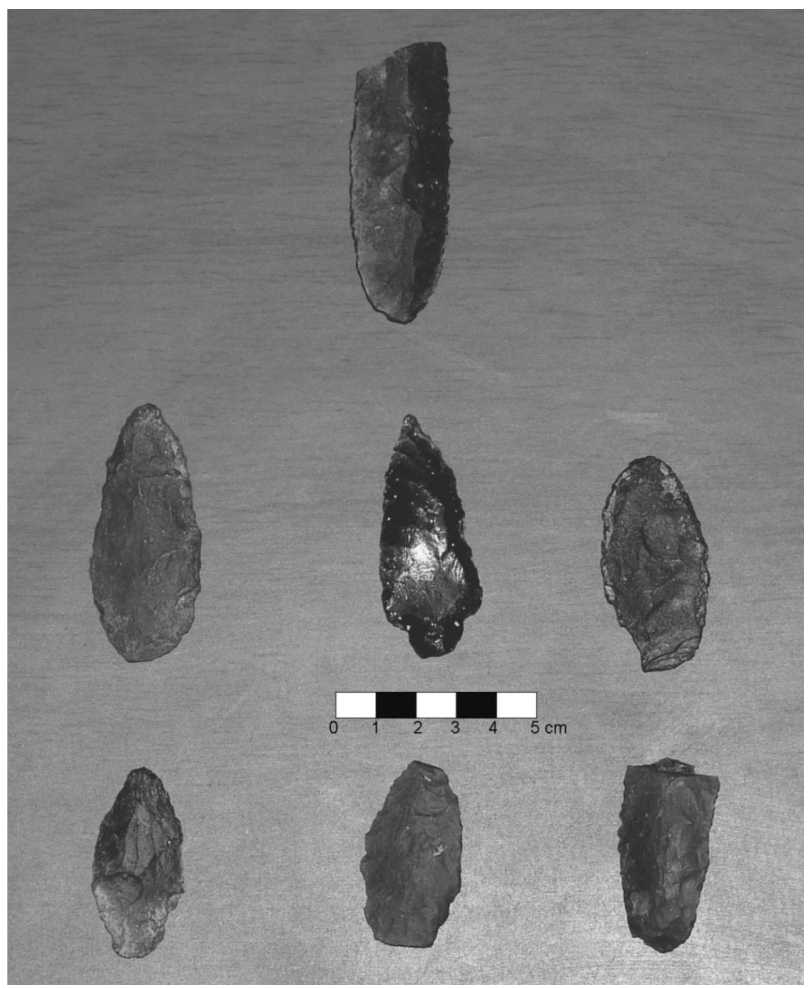

Figura 11. Ejemplares de puntas de proyectil registrados en la prospección realizada en Quebrada Seca.

La prospección en Quebrada Seca, permitió identificar una gran cantidad de estructuras relacionadas a actividades de caza, pero acotadas a un mismo sector. Esto no resulta en la identificación de un único espacio donde esta práctica fue realizada, sino que seguramente los cazadores aprovecharon estos largos cañadones para realizar la cacería, ocultándose detrás de afloramientos rocosos para aguardar a los animales para realizar la cacería. Incluso en algunos de estos cañadones, fueron registradas puntas de proyectil asociadas a grandes bloques rocosos (Jorge Martínez comunicación personal, Mayo 2012).

Por lo tanto, entendemos que más allá de la ausencia de parapetos, los cazadores en Quebrada Seca habrían implementado estrategias semejantes a las identificadas en Antofalla, es decir, aguardar a los animales en su camino ascendente hacia los dormideros, para realizar el ataque cuando el blanco fuera lo más cercano posible. Incluso en el caso de Quebrada Seca, los cañadones constituyen espacios cerrados por los que necesariamente circularían las vicuñas, ya que las conexiones en otros sectores serían imposible por la presencia de los bloques ignimbríticos e incluso, en un caso, se cerró un sector de ascenso a través de la construcción de un pequeño muro cortando un espacio libre entre bloques rocosos, provocando así la circulación obligada por los cañadones.

Por lo tanto podemos concluir que la información obtenida a través del análisis de los paisajes de caza nos habla de ciertos principios generatrices que podrían haber sido implementados por parte de los cazadores en distintos momentos de la historia de ocupación en dos quebradas de la puna catamarqueña. Estos principios se basarían en la posibilidad de aumentar la probabilidad de alcanzar el éxito intentando obtener blancos cercanos 
plausibles de ser alcanzados con las armas con las que contaban las poblaciones locales. En este punto, surge como un aspecto relevante los conocimientos que los cazadores poseen de sus presas para activar estos dispositivos en el momento preciso. Esto significa la puesta en acción de tres aspectos principales asociados al conocimiento. 1) El paisaje. El o los cazadores deben conocer y decidir en qué sector del paisaje se realizará la cacería, utilizando los conocimientos acerca de las presas y sobre los potenciales espacios que éstas seleccionan para ascender. Esto ha quedado claro en la prospección de Quebrada Seca, donde sería irrelevante la preparación de trincheras en diversos lugares, ya que en ningún momento los mismos serían utilizados por las potenciales presas para circular. Por lo tanto las características del paisaje particular juegan un rol relevante a la hora de la materialización de las prácticas de caza, donde los cazadores, conociendo, transformando y experimentando el paisaje lo preparan para el momento del encuentro con las presas. 2) Las presas. Es vital el conocimiento de los movimientos, actitudes y alertas que las tropillas realizan y perciben para poder activar estos espacios. En el caso de Antofalla, los paisajes cinegéticos parecen estar listos para ser activados en el momento de ser necesario, cuando las vicuñas circulen por allí o sean llevadas hacia allí. En el caso de Quebrada Seca, los paisajes se encuentran menos transformados, pero el conocimiento de los cazadores activa también estos espacios en el momento en que sea necesario. 3) Las armas. Más allá del conocimiento técnico sobre la preparación de las armas, aspecto sobre el cual no profundizaremos aquí, los cazadores poseían ciertos conocimientos acerca de las posibilidades del daño que podrían provocar las armas al atacar a una tropilla, implementando o seleccionando ciertos espacios o aumentando la participación de un mayor número de cazadores para aumentar las posibilidades del éxito.

A lo largo de este trabajo hemos propuesto una serie de aportes teóricos, metodológicos y técnicos en torno al concepto de paisaje, específicamente en lo que se refiere a los espacios utilizados para la realización de las prácticas de caza en la puna catamarqueña. Esta información nos ha permitido interpretar algunas características básicas de las estrategias de caza, que posiblemente hayan sido diferentes en cada caso, pero que comparten principios básicos, en los que el objetivo de los cazadores era anticipar y adelantarse a las decisiones tomadas por los animales y de esta manera aumentar considerablemente las posibilidades de alcanzar el éxito (Haber 2003a, 2003b, 2007, 2009; Haber y Moreno 2008; Moreno 2010, 2011b, 2012).

Hemos mostrado que el paisaje por sí solo no nos brinda información relevante, sino que en la interacción de diversas fuentes de información se encuentra la clave de la comprensión de las características de dicho paisaje. Y esta relevancia se enmarca en las relaciones que se construyen, reproducen y modifican entre los agentes humanos y no humanos y que pueden ser observadas a través de marcaciones materiales plausibles de ser identificadas mediante múltiples metodologías arqueológicas.

De igual manera, resulta claro que el sustrato natural en el cual se desarrollan estas prácticas juega un rol importante en la elección de las estrategias de caza, formando parte del interjuego entre los distintos factores sobre los que hablamos al principio del trabajo. De esta manera personas, topografías, animales, rocas, agua forman parte de una continua y rutinaria interacción que da sentido a las prácticas sociales y a las elecciones particulares. 
Agradecimientos: La prospección en Quebrada Seca fue realizada gracias a la financiación del proyecto PIP-CONICET dirigido por Mariana Mondini y Jorge Martínez. Agradezco a ellos y a Carlos Aschero y Salomón Hocsman por la colaboración y apoyo en las tareas de campo y de laboratorio. Quiero agradecer especialmente a Carlos Barot por la imprescindible ayuda en las tareas de campo. A Sebastián Pastor por la invitación para escribir este artículo. Por últimos a los evaluadores cuyos comentarios permitieron enriquecer y profundizar las ideas.

\section{Notas}

1 Seleccionamos tomar la información acerca de las vicuñas, debido al cúmulo de información etológica con la que contamos sobre esta especie, así como también por la posibilidad que otorga la identificación taxonómica mediante osteometría de esta especie frente a llamas y guanacos. 2 Para más información sobre la construcción de estos tipos ver Moreno (2010, 2011a).

3 Estos sectores acotados fueron recorridos en su totalidad para tratar de obtener la mayor cantidad de información posible, obviando así la posible pérdida que presenta la metodología de transectas lineales.

\section{Bibliografía citada}

Anschuetz, H., R. Wilshusen y C. Scheick, 2001 An archaeology of landscapes: Perspectives and directions. Journal of Archaeological Research 9(2): 152-197.

Aschero, C.

1975 Ensayo para una clasificación morfológica de artefactos líticos aplicada a estudios tipológicos comparativos. Informe presentado al Consejo Nacional de Investigaciones Científicas y Técnicas (CONICET). Ms.

1983 Ensayo para una clasificación morfológica de artefactos líticos. Apéndice A y B. Cátedra de Ergología y Tecnología. Facultad de Filosofía y Letras. Universidad de Buenos Aires. MS.

Aschero, C., D. Elkin y E. Pintar.

1991 Aprovechamiento de recursos faunísticos y producción lítica en el precerámico tardío. Un caso de estudio: Quebrada Seca 3 (Puna Meridional Argentina). Actas del XI Congreso Nacional de Arqueología Chilena, tomo II: 101-114. Museo Nacional de Historia Natural, Santiago de Chile.

Aschero, C., L. Manzi y A. Gómez

1993-94 Producción lítica y uso del espacio en el nivel 2b4 de Quebrada Seca 3. Relaciones de la Sociedad Argentina de Antropología XIX: 191-214.

Aschero, C. y J. Martínez

2001 Técnicas de caza en Antofagasta de la Sierra, Puna meridional, Argentina. Relaciones de la Sociedad Argentina de Antropología XXVI: 215-241.

Bender, B.

1993. Introduction: Landscape - Meaning and Action. Landscape: Politics and perspectives (ed. por B. Bender), pp. 1-18. Berg, Oxford.

Bonacic, $\mathrm{C}$.

2005 Vicuña ecology and management. International Camelid Quarterly 4(4): 75-82. 
Bourdieu, P.

1977 Outline of a theory of practice. Cambridge University Press, Cambridge.

1988 Cosas dichas. Gedisa, Barcelona.

Bourdieu, P y L. Wacquant.

1995 Respuestas. Por una antropología reflexiva. Grijalbo, México.

Criado Boado, $\mathrm{F}$.

1993 Límites y posibilidades de la arqueología del paisaje. Spal 2: 9-55.

1999 Del Terrenoal Espacio: planteamientos y perspectivas para la arqueología del paisaje. CAPA 6:1-82.

Criado Boado, F.; Bonilla Rodríguez, A.; Cerqueiro Landín, D.; Díaz Vázquez, M.; González Méndez Fernández, F.; Penedo Romero, R. y E. Rodríguez Puentes

1991 Arqueología del paisaje en Galicia. Arqueoloxia/investigación 6. Santiago. Dirección Xeral de Patrimonio, Consellería de Cultura e Xuventude.

Curtoni, R.

2007 Arqueología y paisaje en el área centro-este de La Pampa. Tesis de Doctorado. Universidad Nacional de La Plata, La Plata.

De Souza, P.

2004 Tecnologías de proyectil durante los períodos Arcaico y Formativo en el Loa Superior (Norte de Chile) a partir del análisis de puntas líticas. Chungara, Volumen especial, tomo I: 61-76.

Elkin, D.

1992 Disponibilidad y explotación de recursos en relación al sitio acerámico Quebrada Seca 3, Antofagasta de la Sierra, Puna de Catamarca. Shincal 2: 1-14.

1996 Arqueozoología de Quebrada Seca 3: indicadores de subsistencia temprana, en la Puna meridional argentina. Tesis de Doctorado. Universidad de Buenos Aires, Buenos Aires.

Escola, P.

1987 Las puntas de proyectil del formativo en Puna y Quebradas de Acceso: un estudio tecno-tipológico de cuatro casos de análisis. Tesis de Licenciatura. Universidad de Buenos Aires, Buenos Aires. 2000 Tecnología lítica y sociedades agropastoriles tempranas. Tesis de Doctorado. Universidad de Buenos Aires, Buenos Aires.

Fernández Distel, A.

1986 Las cuevas de Huachichocana, su posición dentro del precerámico con agricultura incipiente del Noroeste Argentino. Beitrage zur Allgemeinen und Vergleichenden Archaeologie, band 8: 353-430. Manz am Rehein.

Franklin, W.

1982 Biology, Ecology, and Relationship to man of the South American Camelids. Mammalian Biology in South American (ed. por M. Mares y H. Genoways), pp. 457-490. University of Pittsburgh Press, Pittsburgh.

Giddens, A.

1984 The constitution of society. Outline of the theory of structuralism. University of California Press, Berkeley y Los Ángeles. 
Haber, A.

2001 La domesticación del oasis. Actas del XIII Congreso Nacional de Arqueología Argentina 1: 451-466. Córdoba.

2003a Hunting after Domestication. Trabajo presentado en CHAGS 9, Edimburgo.

2003b Informe presentado a la National Geographic Society. Ms.

2006 Una arqueología de los oasis puneños. Domesticidad, interacción e identidad en

Antofalla. Primer y segundo milenios d.C. Jorge Sarmiento Editor, Universitas Libros, Córdoba. 2007 Vicuñas and domesticity. Taphonomy and zooarchaeology in Argentina (ed. por M. Gutiérrez, L. Miotti, G. Barrientos, G. Mengoni Goñalons y M. Salemme), pp. 59-70. BAR International Series 1601, Oxford.

2009 Animism, Relatedness, Life: Post-Western Perspectives. Cambridge Archaeological Journal 19(3): 418-430.

Haber, A. y E. Moreno

2008 Dos veces en la misma trampa. Notas de arqueología casi crepuscular. Jornadas de Arqueología del Área Puneña de los Andes Centro-Sur. Tendencias, variabilidad y dinámicas de cambio (ca. 11000-1000 AP). Horco Molle, Tucumán.

Hocsman, S.

2006 Producción lítica, variabilidad y cambio en Antofagasta de la Sierra-ca. 5500-1500 AP-. Tesis de Doctorado. Universidad Nacional de La Plata, La Plata.

Hoffmann, E., Otte, K., Ponce, C. y M. Ríos

1983 El manejo de la vicuña silvestre. Tomo II. Eschborn. Sociedad Alemana de Cooperación Técnica (GTZ).

Ingold, $\mathrm{T}$.

1993 The temporality of the landscape. World Archaeology 25: 152-174.

Koford, C.

1957 The vicuña and the puna. Ecological monographs Vol. 27 No2. Museum of Vertebral Zoology. University of California.

Lema, C.

2004. Tebenquiche Chico en los siglos XVI y XVII. Tesis de Licenciatura. Universidad Nacional de Rosario, Rosario.

López, G.

2003 Pastoreo y caza de camélidos en el Temprano de la Puna de Salta: Datos osteométricos del sitio Matancillas 2. Intersecciones en Antropología 4: 17-27.

2006 Resultados del análisis arqueofaunístico de un basural, un recinto y un sector interrecinto del sitio Matancillas 2, Puna de Salta. Intersecciones en Antropología 7:207-216.

Martínez, J.

2003 Ocupaciones humanas tempranas y tecnología de caza en la microrregión de Antofagasta de la Sierra (10000-7000 AP). Tesis de Doctorado. Universidad Nacional de Tucumán, Tucumán.

Moreno, E.

2010 Arqueología de la caza de vicuñas en el área del Salar de Antofalla, Puna de Atacama. Una aproximación desde la arqueología del paisaje. Tesis de Doctorado. Universidad Nacional de La Plata, La Plata. 
2011a Tecnología de caza en la quebrada de Antofalla. Revista del Museo de Antropología 4: 17-32. $2011 \mathrm{~b}$ The construction of hunting sceneries. Interactions between humans, animals and landscape in the Antofalla valley, Catamarca, Argentina. Journal of Anthropological Archaeology 31: 104-117.

2012 Propiciando el encuentro. La estructuración de los paisajes de cacería en el contexto andino. Intersecciones en Antropología 13: 327-343.

Moreno, E. y C. Revuelta

2010 La caza de vicunas en Tebenquiche Chico (Dpto. Antofagasta de la Sierra, Catamarca). Un acercamiento de larga duración. Relaciones de la Sociedad Argentina de Antropología XXXV:171-193.

Núñez, L.

1983 Paleoindio y arcaico en Chile: Diversidad, secuencias y procesos. Cuicuilco, México

Núñez, L y M. Santoro

1988 Cazadores de la puna seca y salada del área centro-sur andina (norte de Chile). Estudios Atacameños 9: 11-60.

Olivera, D.

1997 La importancia del recurso Camelidae en la Puna de Atacama entre los 10.000 y 500 años A.P. Estudios Atacameños 14: 29-41.

Olivera, D. y D. Elkin

1994 De cazadores y pastores: El proceso de domesticación de Camélidos en la Puna Meridional Argentina. Zooarqueología de Camélidos. Perspectivas teóricas y metodológicas $\left(1^{\text {a }}\right.$ parte) vol. 1: 95-124. Grupo de zooarqueología de camélidos. Buenos Aires.

Pintar, E.

1996 Prehistoric Holocene adaptations to the Salt Puna of Northwest Argentina. Tesis de Doctorado. Southern Methodist University, Dallas.

Potter, J.

2004 The creation of person, the creation of place: hunting landscape in the American Southwest. American Antiquity 69(2): 322-338.

Quesada, M.

2007 Paisajes agrarios en el área de Antofalla. Procesos de trabajo y escalas sociales de la producción agrícola. Primer y segundo milenio d.C. Tesis de Doctorado. Universidad Nacional de La Plata, La Plata.

Ratto, N.

1994 Funcionalidad versus adscripción cultural: cabezales líticos de la margen norte del estrecho de Magallanes. Arqueología de Cazadores-recolectores. Límites, casos y aperturas (ed. por J. Lanata y L. Borrero), Arqueología Contemporánea 5: 105-120. Buenos Aires.

2003 Estrategias de caza y propiedades del registro arqueológico en la Puna de Chaschuil (Departamento de Tinogasta, Catamarca, Argentina). Tesis de Doctorado. Universidad de Buenos Aires, Buenos Aires.

Revuelta, C.

2005 Apropiación social y vicuñas. Análisis zooarqueológico de la unidad doméstica TC1. Oasis de Tebenquiche Chico - Primer milenio d.C. Tesis de Licenciatura. Universidad Nacional de Catamarca, Catamarca. 
Rodríguez, M.

1998 Arqueobotánica de Quebrada Seca 3: recursos vegetales utilizados por cazadores-recolectores durante el arcaico en la Puna Meridional Argentina. Tesis de Doctorado. Universidad de Buenos Aires, Buenos Aires.

Shott, M.

1997 Stone and shafts redux: the metric discrimination of chipped-stone dart and arrow points. American Antiquity 62 (1): 86-101.

Thomas, D.

1978 Arrowheads and atlatl darts: how the stones got the shaft. American Antiquity 43: 461-472.

Thomas, J.

1996 Time, culture and identity. An interpretative archaeology. Routledge, Londres.

2001 Archaeologies of place and landscape. Archaeological Theory Today (ed. por I. Hodder), pp: 165-186. Polity Press, Cambridge.

Tilley, M.

1994 A phenomenology of landscapes. Places paths and monuments. Berg Publishers, Londres.

Tomka, S.

1992 Vicuñas and Llamas: Parallels in Behavioral Ecology and Implications for the Domestication of Andean Camelids. Human Ecology 20(4): 407-433.

Wheeler, J.

2006 Historia natural de la vicuña. Investigación, conservación y manejo de vicuñas (ed. por B. Vilá), pp: 25-36. Proyecto MACS, Universidad Nacional de Luján, Luján.

Yacobaccio, $\mathrm{H}$.

1988 Camélidos, domesticación y tamaño de la muestra. Reflexiones sobre la utilización animal prehistórica en los Andes. De procesos, contextos y otros huesos (ed. por A. Haber y N. Ratto), pp: 91-100. Facultad de Filosofía y Letras, UBA.

2001 Cazadores complejos y domesticación de camélidos. El uso de los camélidos a través del tiempo (ed. por G. Mengoni, D. Olivera y H. Yacobaccio), pp: 261-282. Ediciones del tridente, Buenos Aires.

Yacobaccio, H y C. Madero.

1992 Zooarqueología de Huachichocana III (Jujuy, Argentina). Arqueología 2: 149-188.

Yacobaccio, H., D. Elkin y D. Olivera.

1994 ¿El fin de las sociedades cazadoras? El proceso de domesticación animal en los Andes Centro Sur. Arqueología de cazadores-recolectores. Limites, casos y aperturas (ed. por J. Lanata y L. Borrero). Arqueología Contemporánea 5: 23-32. Buenos Aires.

Yacobaccio, H., C. Madero, M. Malmierca y M. Reigadas.

1997-98.Caza, domesticación y pastoreo de camélidos en la Puna Argentina. Relaciones de la Sociedad Argentina de Antropología XXII-XXIII: 389-418i 